\title{
El sentido de la escuela en Chile. La creación de paradigmas antagónicos a partir del discurso de política pública, el discurso académico y la investigación educativa
}

\author{
The meaning of the school in Chile. The creation of opposed paradigms from the \\ discourse of the public policy, academia and educational research
}

\author{
Pablo Neut Aguayo ${ }^{a}$, Pablo Rivera Vargas ${ }^{b}$, Raquel Miño Puigcercós $^{c}$ \\ ${ }^{a}$ Facultad de Educación, Universidad de Barcelona. \\ Correo electrónico: paneut@uc.cl \\ ${ }^{b}$ Departamento de Didáctica y Organización Educativa (DOE), \\ Universidad de Barcelona \\ Correo electrónico: pablorivera@ub.edu \\ c Departamento de Didáctica y Organización Educativa (DOE), \\ Universidad de Barcelona \\ Correo electrónico: rmino@ub.edu
}

\begin{abstract}
RESUMEN
Desde el retorno a la democracia se ha reproducido un diagnóstico que informa sobre la crisis del sistema escolar chileno. El objetivo del artículo es analizar la consistencia de dicho diagnóstico en una de sus dimensiones estructurantes: la denominada 'crisis de sentido'. Para ello se revisan tres tipos de aproximaciones desarrolladas en torno a esta problemática: el discurso de política pública, el discurso académico y la investigación educativa. Constatamos que mientras los dos primeros confirman el diagnóstico crítico, las investigaciones empíricas lo desmienten. Dicha situación revela la hegemonía que ha adquirido un determinado paradigma interpretativo en el análisis del sistema escolar y las dificultades para articular, desde este, las distintas dimensiones del fenómeno educativo y los procesos de producción del sentido escolar. Para superar esta limitación, se propone reposicionar el saber que emerge de la experiencia de los actores escolares mediante el desarrollo de una investigación comprometida con la misma.
\end{abstract}

Palabras clave: Crisis de sentido, política de sentido, escolarización, políticas públicas, experiencia escolar

\begin{abstract}
Since the transition to democracy onwards it has been generated a diagnostic reporting about the crisis of the secondary educational system in Chile. The objective of this article focuses on analyzing the consistency of that diagnostic in one of its main dimension: the so-called "crisis of meaning". In order to do that this research examines three approaches related to this issue: discourses from public policies, academia and empirical research. We find that while the first two discourses confirm the critical diagnosis, the conclusions in the empirical research reject this idea. These finding put in perspective the hegemony that an interpretative paradigm has gained in the analysis of the educational system and the difficulties to link the different dimensions in this matter and the process of creation of the educational meaning. To avoid this limitation, this study proposes to highlight the experience of the main participants of the educational system based on a research focused on the relevance of that experience.
\end{abstract}

Key words: Crisis of meaning, politics of meaning, schooling, public policy, schooling experience 
EL SENTIDO DE LA ESCUELA EN CHILE. LA CREACIÓN DE PARADIGMAS ANTAGÓNICOS A PARTIR DEL DISCURSO DE POLÍTICA PÚBLICA, EL DISCURSO ACADÉMICO Y LA INVESTIGACIÓN EDUCATIVA

\section{INTRODUCCIÓN}

Desde el retorno a la democracia se ha reproducido un diagnóstico "de arrastre" que informa sobre la crisis del sistema de educación secundaria en Chile. En efecto, recién comenzado el proceso de transición, académicos asociados al campo oficialista -algunos de los cuales ocuparon prominentes posiciones en el diseño de las políticas públicas posteriores- señalaban el carácter crítico de este nivel de enseñanza (Cariola y Cox, 1991; Lemaitre, 1992; Mena y Rittershaussen, 1991).

A pesar de una inicial oposición ${ }^{1}$, con los años se plegarían a este diagnóstico los sectores de la derecha política, los principales centros de pensamiento o think tank nacionales, los gremios empresariales, el Colegio de Profesores e incluso la Iglesia Católica (Neut, 2018). Promediando la década de 1990, por lo tanto, se verificó un consenso general sobre la crisis de la educación secundaria chilena y la necesidad de llevar a cabo una reforma en los más variados ámbitos del campo escolar (el currículum, el marco institucional, la estructura de los ciclos de enseñanza, la formación profesional, las relaciones pedagógicas, etc.).

A pesar de su carácter consensual y su estatus de "política de Estado" (Cox, 2003) esta intervención no generó la clausura del juicio crítico. Por el contrario, al comenzar el nuevo milenio este adquirió un renovado vigor, ahora enarbolado por los propios actores educativos, quienes, en un alto grado, impugnaron el consenso previo de los sectores políticos y empresariales dirigentes junto a los principios político-ideológicos rectores que habían orientado la reforma de los años noventa. En sucesivas "oleadas manifestativas" entre cuyas expresiones destaca el "mochilazo" del 2001, la "revolución de los pingüinos" verificada el 2006 y el ciclo de movilizaciones vigente que se inicia el año 2011- el movimiento estudiantil -y docente- ha insistido, bajo la premisa de la crisis, en la necesidad de transformar integralmente el modelo educativo nacional (Bellei, Cabalín y Orellana, 2014; Bellei y Cabalin, 2013; Redondo y Muñoz, 2009)

Así, pues, desde 1990 hasta la actualidad la escuela chilena ha sido posicionada en la discusión pública a través de un juicio que señala sus déficits y dificultades. En este contexto, el objetivo del presente trabajo es analizar la consistencia de dicho juicio, específicamente en una de sus dimensiones estructurantes: la denominada "crisis de sentido".

Al respecto, proponemos que existen tres tipos principales de aproximación a esta problemática: el discurso de política pública, el discurso académico y la investigación empírica. Estos tres tipos se articulan en dos grandes paradigmas o matrices interpretativas. La primera matriz es aquella que confirma la crisis de sentido, entendiéndola como un efecto de las condiciones estructurales presentes en el campo educativo. En esta perspectiva se inscriben tanto el discurso de política pública como gran parte del discurso académico. Una segunda matriz, integrada por las investigaciones empíricas, concibe el sentido como el resultado de un proceso de producción de los sujetos educativos. Desde esta posición, lo que se verifica es un fenómeno de pluralización de los sentidos escolares.

El carácter antitético de ambas matrices impide realizar una generalización respecto de la crisis de sentido de la escuela chilena. Dicho diagnóstico, en consecuencia, antes que

Como lo ha demostrado la historia social de la educación, los primeros intentos reformistas se enfrentaron a la oposición de los sectores que previamente habían apoyado la dictadura cívico-militar (la derecha política), sus centros de pensamiento (como Libertad y Desarrollo), los principales gremios empresariales (en particular la Cámara de la Producción y el Comercio, CPC) y un sector de la iglesia católica. Tal es el caso, por ejemplo, del "currículum fracasado" de 1992 (Neut, 2018). 
mostrar una realidad 'en sî', da cuenta de la hegemonía que ha adquirido un determinado paradigma interpretativo en el análisis del sistema escolar chileno.

En términos expositivos, el artículo está dividido en dos grandes secciones. En la primera, y desde una perspectiva teórica general, exploramos algunos de los vínculos existentes entre el proceso de escolarización y el de producción de sentido. Posteriormente, en la segunda y principal sección, verificamos de manera crítica el modo en que ha sido abordado este tema, tanto desde el discurso de la política pública y el académico como desde la investigación educativa en Chile. En este último caso, nos centraremos específicamente en las investigaciones desarrolladas en torno a los estudiantes. En las consideraciones finales analizamos los desafíos que deben afrontar estas aproximaciones, considerando los límites evidenciados en la primera parte.

Esto, y a modo de justificación de la relevancia de la problemática escogida, bajo el supuesto de que una escolarización dotada de sentido por parte de quienes son sus principales 'destinatarios' -los estudiantes- es indispensable para el desarrollo de una educación significativa y relevante a nivel personal y social (Molina, 2008). Del mismo modo, lo es para la consolidación de un sistema que tienda a elevar los niveles de calidad (Molina y Oliva, 2015; Prieto, 2005) y equidad educativa (Neut y Miño, 2018).

\section{LA ESCOLARIZACIÓN COMO POLÍTICA DE SENTIDO}

Como señalan Duschatsky y Corea (2011) "las formas de producción de la subjetividad no son universales ni atemporales sino que se inscriben en condiciones sociales y culturales específicas" (p. 21). Durante los siglos XIX y XX, si no el principal, uno de los más importantes dispositivos diseñado para esta función fue la escuela. Esta, por tanto, se erigió como una institución privilegiada en la constitución de la subjetividad moderna y en la configuración del lazo social² (Dubet, 2007; 2006; Lewkowicz y Corea, 2011; Noyola, 2000).

Dicho ejercicio de producción de la subjetividad supone, junto a la socialización o la adscripción a normas que vehiculizan la cohesión social (Durkheim, 1976), un proceso de reproducción de sentido. Este, de acuerdo con Berger y Luckmann, poseería un carácter multiestratificado. En sus palabras:

Las aprehensiones puramente subjetivas son el fundamento de la constitución de sentido: los estratos más simples del sentido pueden crearse en la experiencia subjetiva de una persona. Los estratos superiores y una estructura más compleja del mismo dependen de la objetivación del sentido subjetivo en la acción social (Berger y Luckmann, 1996, p. 6).

Para los autores, "las reservas de sentido socialmente objetivado y procesado son 'mantenidas' en depósitos históricos de sentido y 'administradas' por instituciones”'

Lewkowicz (2008) define de la siguiente manera el concepto de lazo social: "Llamaré lazo social a la ficción eficaz de discurso que hace que un conjunto de individuos constituya una sociedad. Y a la vez, a la ficción social que instituye los individuos como miembros de esa sociedad. Porque no es la misma experiencia de sí mismo, de la sociedad, del mundo, si uno está instituido como polités ateniense, como laborator medieval, como burgués gentilhombre, como camarada soviético, como ciudadano, o como consumidor, o pariente en una red extendido de parentesco. El lazo, entonces, es la institución de una sociedad para los individuos, pero es también la institución del tipo de individuos pertinentes para esa sociedad" (p. 56). 
(Berger y Luckmann, 1996, p. 10). En estas, continúan, "los sistemas de sentidos que son retenidos quedan recortados en una forma apropiada para su transmisión a las generaciones futuras" (p. 7). Determinadas instituciones, por tanto, constituirían verdaderas "reservas de sentido", objetivados y dispuestos para su adopción por parte de quienes se encuentran sujetos o afectos a su accionar. Particular relevancia adquirirían aquellas destinadas a la transmisión hacia 'las generaciones futuras'. En este registro, la escuela sería, por su naturaleza, una de las principales "instituciones cuya labor incluye el procesamiento social de sentido" (Berger y Luckmann, 1996, p. 9). En concreto, la escuela, en tanto institución dispuesta para la configuración del sujeto, tendría entre sus funciones centrales la de proveer sentido y resguardar que este sea efectivamente alojado en la subjetividad de los estudiantes. Desde esta perspectiva, la escolarización puede ser entendida como un proceso de inscripción de sentido.

Ahora bien, el contenido del mismo y sus procesos de definición resultan de la pugna entre distintos proyectos y sectores sociales, los que traducen sus lógicas, intereses e ideales en discursos pedagógicos. En efecto, como han destacado diversos autores, los "discursos pedagógicos" (Bernstein, 1996), las "gramáticas" del pensamiento pedagógico (Larrosa, 2004) o sencillamente las "pedagogías" (Ayuste y Trilla, 2005) poseen intrínsecamente un contenido normativo que, aunque generalmente se invisibiliza y opera en el plano de lo tácito o subyacente, sanciona y estatuye un sistema de valores a través de los cuales se significa la escolaridad. En palabras de Duschatsky, Farrán y Aguirre (2010), “ese sistema de valores universal trasciende las diferentes situaciones que transitamos, y establece un sentido, un único modo de significarlas, consustancial con su lógica" (p. 84).

De esta manera, los distintos discursos pedagógicos asignan o atribuyen un sentido particular a la experiencia escolar y a los procesos de escolarización, situación que impregna a los sistemas educativos de un conjunto de significaciones prestablecidas y normativizadas para orientar el proceso formativo. Desde esta mirada, pues, la escuela se encontraría "saturada de sentido" (Duschatsky, 2017), muchos de ellos antitéticos y en una relación de lucha por la hegemonía cuya definición excede el marco propio de las relaciones escolares.

En esta línea, Berger y Luckmann (1996) reconocen que los sentidos "objetivados" $-\mathrm{y}$, por lo tanto, validados para su transmisión institucional- "son en buena medida determinados por las relaciones sociales dominantes" (p. 7). Es igualmente este fenómeno el que distingue Bourdieu en su estudio del sistema escolar francés. Para este, la escolarización corresponde a un proceso de imposición de significaciones -que nosotros proyectamos a la noción de sentido- basada en la naturalización de la cultura de los grupos dominantes como cultura escolar "objetiva" y su introyección en la subjetividad de los sectores subalternos (la denominada violencia simbólica), imposición que respondería a las posiciones de poder en el campo educativo (Bourdieu y Passeron, 1995) 3 .

En el fondo, la definición del sentido de la escuela se encontraría subordinada a las relaciones sociales y a la correlación de fuerzas en el campo socioeducativo. Es en este espacio donde se dirimen los sentidos atribuidos por los distintos discursos pedagógicos

\footnotetext{
Será esta lectura de base culturalista la que abrirá el campo de discusión sobre las "políticas de sentido" y la pugna por las significaciones en la escuela. Así, la pedagogía crítica confrontará los "sentidos oficiales" con aquellos antiescolares o contrahegemónico que orientan la disposición hacia la institución educativa de los sectores subalternos (Giroux, 2004; Willis, 2017). A su vez, desde los estudios del currículum se pondrá énfasis en la selección de las significaciones y las formas ideológicas "encubiertas" que impone el proceso de escolarización (Apple, 1991)
} 
y se sancionan aquellos que actuarán a nivel oficial, impregnando normativamente las formas de significación de la escuela. Esta disputa, antes que servir a quienes defienden y caracterizan a la misma como una institución "neutra" -transmisora de un saber objetivo y universal-, expresa el carácter de la escolarización como una política de sentido.

El objetivo del siguiente acápite es precisamente acercarnos a tal política, indagando en los discursos públicos desarrollados en el contexto nacional y contrastándolos con las investigaciones empíricas elaboradas en este ámbito. Lo que pretendemos es explorar las distintas aproximaciones que existen en torno al problema del sentido de la escuela en Chile.

\section{EL SENTIDO DE LA ESCUELA EN CHILE}

La cuestión del sentido de la escuela en Chile ha impregnado los discursos públicos y pedagógicos desde, al menos, la década de 1990. Esta discusión ha posibilitado la emergencia de paradigmas o matrices interpretativas que, más allá de sus particularidades, mantienen una lógica comprensiva y una estructura epistemológico-política compartida.

Al respecto, detectamos dos matrices principales. La primera, de la cual se nutren el discurso de política pública y una parte significativa del discurso académico, tiende a considerar el sentido como un resultante de las condiciones materiales del sistema educativo. En sus antípodas, el otro paradigma aglutina a un conjunto de investigaciones que indagan empíricamente los sentidos construidos por los propios actores educativos en torno a sus procesos de escolarización. Desde esta rivera analítica, antes que un efecto, el sentido es concebido como un ejercicio de producción enmarcado en la experiencia históricamente situada de los sujetos.

\subsection{CRISIS DE SENTIDO DE LA ESCUELA CHILENA: APROXIMACIONES DESDE EL DISCURSO DE POLÍTICA PÚBLICA Y EL DISCURSO ACADÉMICO}

La primera de las matrices señaladas instituye una lógica interpretativa que supone una determinación directa del sistema educativo sobre la experiencia de los actores y sus modos de significación de la escolaridad. En tal paradigma, la crisis de sentido es considerada como un 'efecto residual' de las estructuras de dicho sistema (Neut y Miño, 2018). Tanto el discurso de política pública como gran parte del discurso académico participan de esta matriz y reproducen su lógica interpretativa.

\subsubsection{Aproximaciones desde el discurso de política pública}

El discurso de política pública, en este caso educativa, corresponde a aquellas prácticas discursivas que actúan performativamente sobre las formas de comprensión y producción del conocimiento dentro de un campo determinado, sancionando y definiendo el marco de posibilidades y límites del mismo (Ball, 2003; 2013). Como señalan Flores y Sabrero (2011), a consecuencia de su carácter performativo, el discurso de política pública constituye una "práctica 'generadora' de sentido". Por ello, prosiguen, "entendemos las políticas educativas no solo como conjunto de prácticas institucionales -que regulan las relaciones entre actores e instituciones- sino también como prácticas discursivas que 
EL SENTIDO DE LA ESCUELA EN CHILE. LA CREACIÓN DE PARADIGMAS ANTAGÓNICOS A PARTIR DEL DISCURSO DE POLÍTICA PÚBLICA, EL DISCURSO ACADÉMICOY LA INVESTIGACIÓN EDUCATIVA

producen los sistemas de razón” (pp. 324-325). Es dicho 'sistema de razón' -y sus lógicas interpretativas- el que nos interesa evidenciar.

Al respecto, y retomando lo señalado en la introducción, desde el inicio del proceso transicional se articuló un diagnóstico que evaluaba la enseñanza secundaria chilena de manera insatisfactoria (Cariola y Cox, 1991; Lemaitre, 1992; Mena y Rittershaussen, 1991). Fue un diagnóstico que, tras enfatizar en los déficits y carencias de este nivel, permitió impulsar una decidida intervención reformista.

En el caso de la educación media, la principal política pública diseñada para estos efectos fue el Programa de Mejoramiento de la Calidad y la Equidad de la Enseñanza Media (MECE-MEDIA), en uno de cuyos documentos de origen se afirma

la educación media está hoy en una grave crisis de sentido, tanto para la sociedad en su conjunto, como para los jóvenes que acceden a ella. Su estructura y currículos son anacrónicos; sus resultados son de pobre calidad; las oportunidades que ofrecen son altamente inequitativas (Mineduc, 1994, p. 14).

Como se puede apreciar, a comienzos de los años noventa la crisis de sentido fue rápidamente invocada para validar la reforma de la educación secundaria. Su existencia se tornaba incuestionable. Sin embargo, el 'sistema de razón' en que se soportaba tal juicio contenía una premisa implícita: la pérdida de sentido devenía de las condiciones estructurales del sistema educativo.

En efecto, mientras en el documento citado se reconoce que esta se expresa en los sujetos (los jóvenes o la sociedad), dicha expresión se encuentra subordinada a indicadores sistémicos (los pobres resultados académicos, la inequidad de oportunidades). Los jóvenes o la sociedad -y la crisis de sentido que los aquejaría- constituirían la 'respuesta refleja' de un sistema deficitario. Sería allí, pues, donde habría que buscar la consistencia de sentido de la escuela.

Este ‘sistema de razón' adquiere una mayor fortaleza interpretativa en la medida en que el fenómeno es absorbido por una perspectiva que se moviliza académica y políticamente en el orden de la objetividad y la cuantificación. Así, mejorando los resultados académicos -lo que en esta versión significa a priori elevar la calidad-, actualizando los currículums -generalmente por medio de una selección realizada por 'expertos' - y universalizando el acceso se lograría consolidar una escuela con sentido. A medida que mayor es la objetivación de estos indicadores, la operatoria interpretativa es más implacable para el sujeto escolar. Este, y el sentido mismo, quedan relegados a la condición de 'efecto residual' de las estructuras educativas (Neut y Miño, 2018).

La pregnancia de esta lógica interpretativa en el discurso de política pública ha generado una matriz cuya presencia se prolonga hasta el siglo XXI. Un ejemplo de esto se encuentra en el Informe Final del Consejo Asesor Presidencial para la Calidad de la Educación del año 2006, instancia creada por el gobierno como respuesta al consistente movimiento estudiantil desarrollado durante dicho año. En su obertura, el informe contiene un acápite titulado "El sentido de la educación en la hora presente", en el cual se sostiene que

el diseño del sistema educacional, en todos sus niveles, depende del significado que la sociedad asigne a la educación. Por lo mismo, la pregunta acerca del sentido de la educación - ¿Para qué educar?- debe presidir nuestro trabajo (Consejo Asesor Presidencial para la Calidad de la Educación, 2006, p. 58) 
La cuestión del sentido nuevamente constituye uno de los ejes vertebradores del discurso de política pública, mientras que el 'sistema de razón' que lo soporta persevera en su lógica interpretativa. Así, en el postulado inicial se afirma que el sentido emana del 'significado que la sociedad asigne a la educación'. A pesar de ello, y efectuando la misma operación de desplazamiento que revisamos en el primer caso, en lo restante de este acápite se informa que "la educación posee múltiples funciones para la vida colectiva. La educación tiene importantes funciones económicas; posee también funciones de índole política; y, en fin, de índole moral. Un buen diseño del sistema educacional debe tenerlas todas en consideración" (Consejo Asesor Presidencial para la Calidad de la Educación, 2006, p. 59).

Lo que en un inicio era considerado como un ejercicio de significación propio de la sociedad, prontamente, y de manera solapada, resulta subordinado a las funciones sistémicas de la institución educativa. El sentido, pues, desplazado de los sujetos, dependerá del grado de satisfacción de dichas funciones escolares.

No es casual, por lo tanto, que mientras el Consejo afirma -a modo de encabezado de su informe- que el problema del sentido de la educación 'debe presidir nuestro trabajo', en la práctica dicho problema se zanja en menos de 5 páginas, del total de 252 que contiene el informe. Menos lo es el que, tras esta declaración de principios, el documento se ocupe de desarrollar en profundidad los elementos sistémicos del modelo educacional, entre ellos, el marco regulatorio y legal del nivel secundario, los indicadores de la calidad del mismo y la institucionalidad necesaria para fiscalizar su cumplimiento, el sistema de financiamiento, la reestructuración de los ciclos escolares y el currículum.

En tal sentido, la médula del informe trata sobre las estructuras (institucionalidad, financiamiento, etc.) y los indicadores medibles (calidad, ciclos de aprendizaje, etc.) que permitirían dar cuenta de la satisfacción -o no- de las funciones de la escuela. Aquella 'pregunta acerca del sentido que debe presidir nuestro trabajo' señalada por el Consejo, encuentra su respuesta en dicha satisfacción.

De allí que, en esta aproximación, la lógica interpretativa que posiciona al sentido como un 'efecto residual' de las condiciones sistémicas mantiene su hegemonía en tanto ‘sistema de razón'.

\subsubsection{Aproximaciones desde el discurso académico}

El diagnóstico en torno a la crisis de sentido de la escuela chilena no es exclusivo del discurso de política pública. En él también participa una parte significativa de los discursos académicos. Estos constituyen creaciones intelectuales que modulan las perspectivas de análisis de un fenómeno. Cuando tales perspectivas se consolidan y adquieren preeminencia para la comprensión del mismo, se convierten en un discurso interpretativo dominante o DID. Este, en palabras de Touraine (2007), "no es una simple ideología que protege y estimula los intereses del grupo dirigente; es una creación estrictamente intelectual, una representación de la sociedad, de la acción de las instituciones y de las conductas individuales y colectivas" (p. 70).

Dicha representación, al adquirir una posición interpretativa hegemónica, impregna al discurso académico que le había dado origen. De esta manera, los discursos académicos producen y perpetúan un determinado DID con la misma fuerza que este les proporciona su legitimidad, una vez instalado como perspectiva interpretativa hegemónica. 
EL SENTIDO DE LA ESCUELA EN CHILE. LA CREACIÓN DE PARADIGMAS ANTAGÓNICOS A PARTIR DEL DISCURSO DE POLÍTICA PÚBLICA, EL DISCURSO ACADÉMICOY LA INVESTIGACIÓN EDUCATIVA

En Chile, una porción significativa de los discursos académicos sobre el sentido de la escuela comparten el diagnóstico de su crisis, configurando el DID en torno a este fenómeno; no obstante, esta connivencia, difieren respecto de las causas específicas que producirían la crisis de sentido de la escuela chilena. Para algunos, esta derivaría de los cambios culturales impulsados por los procesos de globalización, de internacionalización de los mercados y de producción de nuevas tecnologías, realidades que redefinirían los modos tradicionales de subjetivación y constitución del vínculo social (Hevia, 2006). Otros, desde una perspectiva fenomenológica, la han caracterizado como una manifestación propia de la 'era del vacío', es decir, como un síntoma que expresa la pérdida de los referentes antropológicos y éticos que proporcionarían un lugar y un horizonte comprensivo para la acción individual y social (Flores, 2001).

En ambos casos, la adscripción al 'sistema de razón' que venimos revisando se produce por la subordinación del sujeto a procesos globales que modularían las realidades nacionales, locales y escolares. La crisis de sentido, en esta lógica, constituiría el resultado de las condiciones actuales de producción del sujeto y de constitución del lazo social. De allí que, antes que una situación que ataña exclusivamente a los procesos educativos, la crisis afectaría el sentido de la vida en su totalidad, incluyendo por 'osmosis estructural' a la escuela y quienes se alojan en su seno.

Junto a esta interpretación que concibe la crisis de sentido como el efecto de procesos globales que intervienen en el campo educativo, se desarrolla otro posicionamiento académico que la comprende como el resultado concreto de las particularidades del sistema chileno. La mayoría de quienes participan de esta versión se presentan críticos respecto del discurso de la política pública; sin embargo, sus premisas subyacentes tributan al 'sistema de razón' que este inaugura. En tal rivera analítica, por lo tanto, las estructuras y funciones del sistema -aunque impugnadas por su carácter reproduccionista- nuevamente constituirían el soporte basal de la "crisis de sentido de la educación formal" (Abarca, Hidalgo y Tari, 2012). Un ejemplo paradigmático al respecto lo constituye el análisis de Sánchez y Santis (2009), quienes afirman que

la mercantilización de la educación ha provocado el distanciamiento entre el estado y la sociedad civil, y sobre todo respecto de los jóvenes estudiantes populares, lo cual se expresa en tres crisis de sentido:

1. Crisis de calidad, debido a los magros resultados obtenidos por los establecimientos educacionales municipales y algunos subvencionados en los índices de medición SIMCE, PSU e internacionales (...)

2. Crisis de inequidad, debido a las diferencias de rendimiento entre colegios particulares, subvencionados y municipales (...)

3. Crisis de segmentación social de los establecimientos. Se ha producido un proceso de apartheid educativo entre los establecimientos (pp. 444-445).

Como se evidencia en el extracto, el sujeto se encuentra absolutamente desplazado como eje de comprensión del fenómeno. En la práctica, su participación se restringe a la recepción pasiva de los efectos derivados de las estructuras del sistema. Así, esta operatoria interpretativa reactualiza -ahora desde una perspectiva crítica- las lógicas del 'sistema de razón' propio del discurso de la política pública.

A modo de cierre de este subacápite, es posible constatar la presencia y extensión de una matriz interpretativa que impregna tanto al discurso de política pública como a 
gran parte del discurso académico, aglutinado en torno a un DID específico. Dicha matriz se soporta sobre dos proposiciones centrales. La primera de ellas explícita: la educación secundaria chilena arrastra una perenne crisis de sentido. La segunda, tácita o subyacente: tal crisis deviene de las condiciones estructurales del sistema educativo. El corolario que surge de la articulación de ambas proposiciones es categórico: en la mentada crisis de sentido de la escuela, el sujeto es relegado a la posición de receptáculo inerme de las condiciones sistémicas. Aunque tal crisis se exprese o se encarne en los sujetos, no son estos quienes la producen, ni siquiera los que la informan.

Esto es así, pues, por su misma lógica interna, tal ‘sistema de razón' desestima la concurrencia del juicio de los actores educativos para señalar la crisis de sentido. Esta se desprende inequívoca y unilateralmente de las condiciones estructurales y funcionales del sistema educativo. Al respecto, resulta indicativo que en ninguna de las citas utilizadas en este apartado se haga referencia, se indique, se transcriba o se exprese la opinión directa y concreta de algún estudiante, profesor o directivo. En este aspecto, compartimos la proposición de Molina y Oliva (2015) quienes afirman que

la tendencia general en estos debates es concebir al sujeto como "efecto" o "reflejo" de una estructura o un sistema -educativo en nuestro análisis-, y no como fuente primaria de construcción de esta. En términos sintéticos, en estas concepciones el sujeto no habla y no está en el origen del sentido; por el contrario, es hablado por la ley y la cultura, ya sea para afirmarlo o negarlo/invisibilizarlo (p.1131).

Es en este contexto que una de las metáforas utilizadas por Touraine (2007) adquiere sentido. Para este, el discurso interpretativo dominante de las ciencias sociales ha producido la imagen de "una sociedad vacía" (p. 63) o "una sociedad sin actores, completamente sometida a determinismo externos" (p. 28). A nuestro juicio, el 'sistema de razón' que informa sobre la crisis de sentido de la educación secundaria chilena proyecta la misma imagen de una escuela vacía, es decir, "sin actores y sin sujeto" (Touraine, 2007, p. 46).

\section{2. ¿CRISIS DE SENTIDO DE LA ESCUELA CHILENA?: APROXIMACIONES DESDE LA INVESTIGACIÓN EMPÍRICA}

La escuela vacía que proyecta el tipo de discurso analizado no agota el espectro de aproximaciones en torno al sentido de la escuela chilena. En efecto, esta problemática también ha sido abordada por la investigación empírica, configurando un tipo de acercamiento que difiere del precedente. La diferencia sustancial entre ambas matrices es que, en esta última versión, el 'sistema de razón' se soporta en la valorización de la subjetividad de los actores escolares. Serían estos los que producirían el sentido $-\mathrm{o}$ su crisis- a partir de su experiencia históricamente situada. En otras palabras, antes que deducir una crisis de sentido de la escuela a partir del análisis de las estructuras y funciones del sistema, este tipo de aproximación explora su consistencia indagando directamente en los procesos de significación desarrollados por los actores escolares.

En este punto resulta necesario realizar un ejercicio de delimitación. En primer lugar, debemos señalar que, en este paradigma, el sentido se produce en la experiencia escolar. Sin embargo, no todo estudio centrado en dicha experiencia informa necesariamente sobre el sentido de la escuela. Por ejemplo, mientras las pioneras investigaciones sobre experiencia 
escolar en Chile (Lechner, 1982) -generalmente tributarias de la tradición etnográficaanalizan prácticas y relaciones escolares, poco es lo que señalan sobre los sentidos que los propios actores escolares atribuyen a la misma.

Del mismo modo, existen trabajos cuyo objetivo es indagar en el sentido que los actores escolares atribuyen a dimensiones específicas de la experiencia escolar y no a esta en su conjunto. Destacan al respecto las investigaciones desarrolladas en torno al sentido del aprendizaje (Valenzuela, 2009), de la participación escolar (Prieto, 2005) y de la formación ciudadana y política (Ravelo y Radovic, 2018; Redon, Angulo y Vallejos, 2015), así como aquellas que analizan el sentido de pertenencia a la institución educativa (Castillo, Rodríguez y Escalona, 2018; Quaresma y Zamorano, 2016) y el sentido de futuro (Molina, 2013a) que construyen los estudiantes chilenos a partir de su experiencia escolar.

La razón de estas precisiones es advertir o explicitar que, en esta sección, nuestro foco de análisis estará puesto específicamente en las investigaciones cuyo objetivo es desentrañar el sentido global que construyen los estudiantes secundarios chilenos en torno a la escuela y su experiencia. Dichas investigaciones pueden ser divididas en dos grupos principales que reseñaremos a continuación.

\subsubsection{El sentido de la escuela en jóvenes chilenos con trayectorias 'irregulares'}

El primero de los grupos señalados, minoritario cuantitativamente, aglutina los trabajos que indagan en los sentidos construidos por sujetos con trayectorias escolares 'irregulares', es decir, jóvenes desescolarizados o estudiantes reescolarizados que asisten a las denominadas escuelas de segunda oportunidad.

Para el caso de los jóvenes desescolarizados, Sapiains y Zuleta $(2001 ; 2009)$ han detectado dos grandes núcleos de sentido en torno a la escuela. El primero de ellos se caracteriza por la legitimación de la institución desde una perspectiva instrumental. Esta, pues, representaría el lugar de la integración social y el medio que permitiría a sus estudiantes acceder a mejores condiciones de vida. Como contraparte, un segundo núcleo de sentido se configura desde la impugnación de la institución educativa. En esta perspectiva, la escuela es percibida como un espacio ajeno, alienante, coercitivo y, sin embargo, necesario. En palabras de los autores, "más allá de los muros de la escuela se encuentra el vacío y la deslegitimación social. La escuela y la escolarización se constituyen en el pilar fundamental para ser, en las palabras de los mismos jóvenes entrevistados, «alguien en la vida»" (Sapiains y Zuleta, 2009, p. 47). Es por ello que la impugnación señalada como segundo núcleo de sentido no supone un rechazo absoluto a la escolarización, sino un tipo de acercamiento que la comprende como un "sinsentido necesario" (Sapiains y Zuleta, 2009, p. 54; Salinas y Franssen, 1997). Guerrero y Palma (2010) también han destacado esta relación ambivalente con la institución escolar. Para las autoras, los jóvenes desescolarizados validan instrumentalmente a la institución y resienten lo que ellos viven como procesos de expulsión, pues, mediante estos, "se les deja fuera de la sociedad, de la posibilidad de 'ser alguien en la vida' (como ellos mismos lo dicen)" (Guerrero y Palma, 2010, p. 1036).

Por su lado, la representación de los jóvenes reescolarizados en escuelas de segunda oportunidad es similar a la que producen los desescolarizados. Estos, a partir de una 'racionalidad pragmática', significarían la escuela como una instancia privilegiada en la adquisición de habilidades comunicativas y la obtención de una certificación que pavimentaría su ingreso en el mercado laboral (Espinoza, González, Castillo y Neut, 2018). 
De esta manera, tanto los jóvenes desescolarizados como los reescolarizados producen un sentido en torno a la escuela que es informado por una racionalidad pragmática, pero que -tras la impugnación de la institución y su validación instrumental- expresa un fuerte juicio de valor: la escuela es el espacio para lograr, de acuerdo con las palabras de los mismos jóvenes, "ser alguien en la vida". Así, pues, aunque resistida y criticada, el sentido de la escuela se vincula con su capacidad de hacer advenir un sujeto integrado y validado dentro de la comunidad. Es decir, un sujeto reconocido socialmente como tal.

\subsubsection{El sentido de la escuela en jóvenes chilenos con trayectorias 'regulares'}

Un segundo grupo de investigaciones se aboca a desentrañar los sentidos que construyen los jóvenes con trayectorias escolares 'regulares', es decir, que cursan sus estudios secundarios de acuerdo con la progresión oficial del ciclo de enseñanza.

En estas se revela una escolarización pletórica de sentidos. En efecto, ya desde los primeros estudios se constata la relevancia de la experiencia escolar para los jóvenes y los procesos de significación que esta desencadena. Así, por ejemplo, aunque Salinas y Franssen (1997) distinguen distintos tipos de experiencia escolar - diferenciadas fundamentalmente por la dependencia del establecimiento (municipal, subvencionado o particular), la modalidad educativa (científico-humanista o técnico-profesional) y la escolaridad de los padres- los autores afirman que en general

los jóvenes le asignan un sentido a su experiencia escolar que consagra y confirma el modelo cultural de la escuela (la formación cultural, personal y social), y aunque prepararse para el trabajo y para dar la $\mathrm{PAA}^{4}$ son imperativos de selección del sistema, son desplazados por estas otras dimensiones (Salinas y Franssen, 1997, s/p).

Estudios posteriores confirmarán la existencia de múltiples sentidos escolares, que proporionan una imagen menos "monolítica" que la proyectada en la cita. Es decir, donde la subjetividad juvenil no produce necesariamente una subordinación a un sentido hegemónico.

Molina (2008) distinguirá cuatro figuras significantes entre los estudiantes secundarios que asisten a liceos públicos. Para estos, las principales configuraciones de sentido de la escuela serían: "prepararte para el futuro", "desarrollarte como persona", "tener más cultura" y "vivir la experiencia/etapa". La existencia de dichas figuras revelaría la generación de un horizonte de sentido fragmentado (Molina, 2008; Molina y Oliva, 2015), situación que también ha sido detectada en otros países de la región (Kessler, 2002; Tenti, 2000) y de Europa (Bonal, 2003; Dubet y Martuccelli, 1998). En el caso chileno, dicha fragmentación no descansaría en un criterio de división unívoco, sino que se expresaría aleatoriamente dentro de cada centro educativo, independiente de sus adscripciones socioeconómicas, su composición de género o su modalidad educativa. Por ello, el autor sostiene la existencia de "una polarización de los horizontes de sentido, no tanto entre diferentes liceos, sino al interior de una misma aula o sala de clases" (Molina, 2013b, p. 61).

El proceso de pluralización de los sentidos escolares también ha sido detectado por otros investigadores. Algunos, siguiendo la propuesta de Dubet y Martuccelli (1998), han

Prueba de Aptitud Académica. Evaluación aplicada en Chile hasta el año 2002 como mecanismo de selección para el ingreso a la educación superior. 
señalado que la experiencia escolar -y su sentido- emerge de la articulación subjetiva de distintas lógicas de acción. En esta perspectiva, Rojas (2006) ha identificado dos configuraciones principales en las escuelas chilenas. La primera de ellas, impregnada por una lógica de acción estratégica, supone el desarrollo de un sentido instrumental sobre la institución educativa. La segunda, donde predomina la lógica de la subjetivación, induciría a la formación de un sentido de carácter autoformativo, es decir, donde el sujeto valoriza desde una posición crítica a la escuela, al mismo tiempo que reconoce la capacidad de acción y la autonomía que tiene para forjarse a sí mismo dentro de dicho escenario.

Otras investigaciones han confirmado la relevancia que posee la escuela en "jóvenes urbanos populares" (Moya, 2008). Estos desarrollarían un sentido pragmático en torno a su experiencia escolar y conciben a la institución como un medio para mejorar los niveles de vida. Así, por tanto, "la educación formal cobra sentido para la concreción de proyectos de vida [pues] es significada como 'la' forma de ascenso y promoción social" (Moya, 2008, p. 11). No obstante, a esta racionalidad pragmática se le agregaría una de carácter relacional, que valoriza la escuela en tanto espacio de creación de vínculos humanos. Esta misma situación - la connivencia de sentidos pragmáticos y relacionales- es la que se ha evidenciado en niños que asisten a la educación básica de escuelas rurales (Silva, Bastidas, Calfuqueo, Díaz y Valenzuela, 2013).

La revisión que acabamos de realizar en torno a la investigación empírica permite arribar a dos observaciones generales. La primera de ellas es que, a diferencia del 'sistema de razón' que instaura el discurso de política pública y el académico, desde la experiencia concreta de los estudiantes secundarios, la escuela chilena se encuentra colmada de sentidos. En efecto, sean estos pragmáticos, instrumentales, formativos, relacionales o de cualquier otro tipo, los jóvenes chilenos -escolarizados, desescolarizados o reescolarizados- producen indefectiblemente procesos de significación en torno a la escuela y el modo en que la vivencian. Tales procesos se cristalizan y decantan en matrices comprensivas a través de las cuales se interpreta globalmente el sentido de la escolarización. Por ello, en este 'sistema de razón', la referida crisis de sentido no encuentra respaldo. En otras palabras, la crisis de sentido que estatuye el discurso de política pública y el DID académico resulta inexistente desde la perspectiva de los 'destinatarios' del sistema escolar. Esto, pues, antes que una crisis lo que se evidencia es una proliferación de sentidos escolares.

Al respecto, y como segunda observación general, consideramos que dicha proliferación o pluralización se estructura en base a una tensión central, determinada por la preponderancia que los estudiantes atribuyen al orden instrumental o al relacional dentro de su proceso de escolarización. Ambas racionalidades son movilizadas por los jóvenes al momento de significar su experiencia escolar y producen una 'tensión de sentido' cuya definición corresponde a un ejercicio del sujeto.

En la práctica, cuando prima la racionalidad instrumental, la escuela es concebida como un medio que habilita posibilidades económicas y de promoción social para el futuro, mientras que las alusiones al sentido de la misma -al igual que en el caso de los jóvenes con trayectorias 'irregulares' - se expresan en la fórmula "ser alguien en la vida" (Moya, 2008, p. 9) o alguna de sus derivaciones: "ser alguien" (Salinas y Frassen, 1997, s/p), "ser algo más” (Silva et al., 2013, p. 10), "tener posibilidades de vida” (Baeza, 2001, p. 85), "prepararte para la vida" (Molina, 2008, p. 117).

Por el contrario, cuando la definición se vuelca hacia el orden relacional, lo significativo es la vivencia del presente y el contacto con la otredad. En esta perspectiva, los estudiantes, 
antes que valorizar la escuela por sus consecuencias sociales, lo hacen en tanto espacio de vinculación humana, interacción que para ellos representa la principal instancia formativa. En este registro, el sentido de la escuela se sintetiza en la fórmula "vivir la experiencia/ etapa" (Molina, 2008, p. 116) y se materializa en "el acto de compartir con otros" (Silva, et. al, p. 5) y tener "contacto con otros jóvenes" (Baeza, 2001, p. 85). Como señalan Salinas y Franssen (1997), en este polo de sentido "más que los estudios o los profesores, son las relaciones en el grupo de pares que aparece en el centro mismo de su vida escolar. Este compartir se basa en el sentimiento de una experiencia común" (s/p).

La 'tensión de sentido' que produce la movilización conjunta de ambas racionalidades es un proceso que los mismos estudiantes reconocen, estableciendo una "diferencia entre el asistir a clases y el concurrir al liceo" (Baeza, 2001, p. 91), y con la cual deben lidiar al momento de definir el sentido global de la escuela, definición cuya mera presencia supone un cuestionamiento al diagnóstico que, desde comienzos de la década de 1990, ha afirmado categóricamente la crisis de sentido de la educación secundaria chilena.

\section{DISCUSIÓN Y CONCLUSIONES}

La escuela es una institución donde circulan y se entrecruzan una pluralidad de sentidos. Estos se originan y articulan en distintos niveles. Desde una perspectiva macrosistémica, los múltiples discursos pedagógicos atribuyen un sentido a la escolarización y pugnan por imponer su cariz al sistema en su totalidad. Estos, en un nivel intermedio o mesosistémico, son movilizados por discursos de política pública y académicos que configuran un discurso interpretativo dominante o DID (Touraine, 2007). En el caso chileno, ambos niveles se encuentran alineados en torno a un axioma central: la escuela padecería una crónica crisis de sentido. La extensión y pregnancia de tal diagnóstico da cuenta de la hegemonía que ha adquirido una determinada matriz interpretativa o paradigma en el análisis del sistema escolar chileno.

Sin embargo, bajo este nivel macro y meso se encuentran los sujetos, quienes significan la escolaridad a partir de su experiencia cotidiana. Desde esta posición, la mencionada crisis carece de consistencia y, por el contrario, emerge la imagen de una escolarización colmada de sentidos.

El antagonismo que se evidencia entre ambas matrices o paradigmas impele a revisar distintos aspectos en los tres tipos de aproximación que hemos revisado. Desde la perspectiva de la investigación resulta urgente ampliar el conjunto y las características de los actores escolares estudiados. Por otra parte, el discurso de política pública y el DID académico debe ser sometido a una revisión crítica que permita vincular sus presupuestos con el saber que emerge de la experiencia concreta de dichos actores. En las tres aproximaciones, pues, se presentan desafíos que emanan de sus propias limitaciones o insuficiencias.

\subsection{DESAFÍOS PARA LA INVESTIGACIÓN}

La investigación empírica ha demostrado que los estudiantes chilenos desarrollan múltiples sentidos en torno a la escuela y su experiencia en la misma. No obstante, dicha verificación, del conjunto de trabajos que se inscriben en esta perspectiva emerge una imagen limitada respecto de la problemática en cuestión. 
EL SENTIDO DE LA ESCUELA EN CHILE. LA CREACIÓN DE PARADIGMAS ANTAGÓNICOS A PARTIR DEL DISCURSO DE POLÍTICA PÚBLICA, EL DISCURSO ACADÉMICO Y LA INVESTIGACIÓN EDUCATIVA

Esto es así pues tales investigaciones se caracterizan por trabajar con muestras que, en su sumatoria, presentan una gran concentración territorial y socioeconómica. En efecto, la mayoría de los trabajos se basa en entrevistas a estudiantes de la región Metropolitana de Chile y, particularmente, de Santiago (Baeza, 2001; Molina, 2013b; 2008; Molina y Oliva, 2015; Moya, 2008; Salinas y Franssen, 1997). En la literatura revisada, solo dos investigaciones escapan a este patrón: una de ellas porque tiene un carácter regional y rural y se centra en relatos de estudiantes mapuche que viven en la comuna de Ercilla, región de la Araucanía (Silva et al., 2013); la segunda, debido a que tuvo un alcance nacional (Edwards, Calvo, Cerda, Gómez y Inostroza, 1995) y fue encargada por el Ministerio de Educación con el objetivo de generar insumos para el diseño del mencionado Programa de Mejoramiento de la Calidad y la Equidad de la Enseñanza Media (MECE-MEDIA).

Un segundo eje de concentración es el referido a las características socioeconómicas de los estudiantes que participaron en los respectivos estudios. En la práctica, la mayoría de las investigaciones se ocupa de indagar en el sentido que construyen los jóvenes de estrato socioeconómico bajo (Baeza, 2001; Moya, 2008; Silva et al., 2013) o, en su defecto, que asisten a escuelas públicas municipalizadas (Molina, 2013; 2008; Molina y Oliva, 2015) . En este aspecto, se diferencian las publicaciones que incluyen a estudiantes del sector subvencionado y particular (Edwards, et. al, 1995; Salinas y Franssen, 1997).

La concentración territorial y socioeconómica impide la generación de un panorama general y, a la vez, representativo de las distintas realidades educativas existentes en las escuelas chilenas. Por ello se torna urgente complementar la investigación existente con nuevos trabajos que incluyan a todos los segmentos socioeconómicos -cuestión aún más apremiante considerando el alto grado de segmentación que presenta el sistema chileno (Bellei, 2013; Gallego y Sapelli, 2007; García-Huidobro, 2007)-, que integren a estudiantes provenientes de distintas regiones y que consideren variables como el género, la etnia u otro de los marcadores que la investigación internacional (Bonal, 2003) ha considerado como relevantes para indagar en los procesos de construcción de sentido que desarrollan los estudiantes secundarios en torno a la escuela.

Este ejercicio permitirá reconstituir -a partir de las particularidades socioeconómicas, regionales, étnicas, de género, etc.- un panorama global sobre los sentidos de la escuela en Chile; panorama que, por el momento, está hegemonizado por las significaciones indagadas entre jóvenes de sectores populares de Santiago de Chile.

\subsection{DESAFÍOS PARA EL DISCURSO DE POLÍTICA PÚBLICAY EL ACADÉMICO}

El 'sistema de razón' sobre el que descansa el discurso de política pública y el DID académico emerge de una lógica interpretativa que instaura la invisibilización de la experiencia concreta de los actores escolares. Esta resultaría de las condiciones estructurales y funcionales del sistema y allí, por tanto, habría que buscar la consistencia de sentido de la escuela.

A mayor 'objetivación' estructural (mediante la creación de indicadores medibles de calidad, equidad, eficiencia, etc. y de un discurso que instaura este tipo de aproximación

Si bien es cierto que todos los estudios referenciados en este paréntesis se desarrollaron en escuelas municipales, los autores seleccionaron liceos representativos de distintos estratos socioeconómicos, siguiendo la clasificación oficial elaborada a partir del SIMCE. Como explicitan los mismos, en el sistema educativo chileno no existen colegios municipales clasificados en la categoría de estrato socioeconómico alto. 
como 'el' conocimiento válido), más irrelevante se torna la subjetividad de los actores. Tal enfoque, que puede ser útil para el procesamiento de grandes volúmenes de información -cuestión relevante en el contexto de la creación de un sistema escolar masificado-, demuestra su límite en la contrastación empírica. En otras palabras, los beneficios de un sistema de conocimiento con una gran capacidad estadística se ven contrarrestados por la progresiva distancia que genera con la realidad vivida por los sujetos. De allí que, mientras tal aproximación ha insistido reiteradamente en la crisis de sentido de la escuela chilena, la investigación en torno a estos la ha desmentido.

Así, de existir una crisis, esta sería o de los discursos hegemónicos sobre el sentido de la escuela (que habrían perdido su capacidad instituyente y de inscripción en la subjetividad estudiantil) o de la articulación entre los distintos niveles del fenómeno educativo (producto de la incomunicación entre las atribuciones de sentido generadas en el macro y mesosistema y los sentidos producidos en el nivel micro). Considerando esto último, uno de los principales desafíos del discurso de política pública y del DID académico es rearticular sus vínculos con la investigación educativa, reposicionando la experiencia de los sujetos precisamente allí donde fue invisibilizada por las estructuras. A nuestro juicio, esta operación redituaría en la consolidación de un sistema educativo más inclusivo, democrático y equitativo.

En efecto, si la escuela, en una de sus dimensiones, tiene por objetivo principal la inscripción de sentidos, entonces el proceso de definición de estos adquiere una función estratégica e imprime el carácter al sistema en su conjunto. Por ello, una 'política de sentido' sancionada a partir de un 'sistema de razón" que ignora la experiencia de los sujetos escolares, operará -tal como se viene advirtiendo hace décadas- como un mero instrumento de imposición simbólica y de inculcación arbitraria de significados (Bourdieu y Passeron, 1995). Por el contrario, una 'política de sentido' estatuida e incidida por los procesos de significación que desarrollan los actores escolares, permitiría el despliegue de una educación contextualizada, relevante y significativa para quienes son sus destinatarios (Molina, 2013b; Molina y Oliva, 2015; Neut y Miño, 2018).

Es por ello que, en la definición de los sentidos 'oficiales' de la escuela, se debería incluir de manera privilegiada a aquellos que construye la comunidad educativa en general y los estudiantes en particular. Situación que requiere, como condición de posibilidad, la revalorización de la experiencia de los sujetos escolares y el desarrollo de una investigación comprometida con la misma.

\section{REFERENCIAS BIBLIOGRÁFICAS}

Abarca, P., Hidalgo, F. y Tari, G. (2012). Crisis de sentido de la educación formal: resistencias y construcción de la juventud popular post-dictadura. Extramuros: revista de la Universidad Metropolitana de Ciencias de la Educación, 11, 85-100.

Apple, M. (1991). Ideología y Currículum. Madrid: Akal.

Ayuste, A. y Trilla, J. (2015). Pedagogías de la modernidad y discursos postmodernos sobre la educación. Revista de Educación, 336, 219-248. Última visita 30 de diciembre de 2018. Recuperado desde http://www.ince.mec.es/revistaeducacion/re336/re336_13.pdf

Ball, S. (2003). Profesionalismo, gerencialismo y performatividad. Revista Educación y Pedagogía, 15(37), 85-10. Última visita 05 de diciembre de 2018. Recuperado desde http://aprendeenlinea. udea.edu.co/revistas/index.php/revistaeyp/article/view/5979 
Estudios Pedagógicos XLV, $\mathrm{N}^{\circ}$ 1: 151-168, 2019

EL SENTIDO DE LA ESCUELA EN CHILE. LA CREACIÓN DE PARADIGMAS ANTAGÓNICOS A PARTIR DEL DISCURSO DE POLÍTICA PÚBLICA, EL DISCURSO ACADÉMICO Y LA INVESTIGACIÓN EDUCATIVA

Ball, S. (2013). Performatividad y fabricaciones en la economía educacional: rumbo a una sociedad performativa. Pedagogía y Saberes, 38, 103-113. Doi: 10.17227/01212494.38pys103.113

Baeza, J. (2001). El Oficio de Ser Alumno en Jóvenes de Liceo de Sector Popular. Santiago: Universidad Católica Cardenal Raúl Silva Henríquez.

Bellei, C. (2013). El estudio de la segregación socioeconómica y académica de la educación chilena. Estudios Pedagógicos, 39(1), 325-345. Doi: 10.4067/S0718-07052013000100019

Bellei, C. \& Cabalin, C. (2013). Chilean student movements: Sustained struggle to transform a market-oriented educational system. Current Issues in Comparative Education, 15(2), 108-123. Última visita 02 de diciembre de 2018. Recuperado desde https://www.tc.columbia.edu/cice/ pdf/28175_15_02_Bellei_Cabalin.pdf

Bellei, C., Cabalin, C. \& Orellana, V. (2014). The 2011 Chilean student movement against neoliberal education policies. Studies in Higher Education, 39(3), 426-440. Doi: 10.1080/03075079.2014.896179

Berger, P. y Luckmann, T. (1996). Modernidad, pluralismo y crisis de sentido. Estudios Públicos, $63,1-54$

Bernstein, B. (1996). Pedagogía, control simbólico e identidad. Madrid: Morata.

Bonal, X. (2003) Apropiacions escolars: Usos i sentits de l'educació obligatòria en l'adolescència. Barcelona: Octaedro.

Bourdieu, P. y Passeron, J. (1995). La reproducción. Elementos para una teoría del sistema de enseñanza. Barcelona: Laia.

Cariola, L. y Cox, C. (1991) La educación de los jóvenes: crisis de relevancia y calidad de la educación media. En M. Mena y S. Rittershaussen (Eds.). La juventud y la enseñanza media (pp. 23-42). Santiago: Corporación de Promoción Universitaria.

Castillo, V., Rodríguez, C. y Escalona, J. (2018) Participación, vida democrática y sentido de pertenencia según tipo de establecimiento educativo en Chile. Páginas de Educación, 11(2), 108-129. Doi: 10.22235/pe.v11i2.1630

Consejo Asesor Presidencial para la Calidad de la Educación (2006). Informe Final. Última visita 20 de noviembre de 2018. Recuperado desde http://unicef.cl/web/informe-final-del-consejo-asesorpresidencial-para-la-calidad-de-la-educacion/

Cox, C. (2003). Las políticas educacionales de Chile en las últimas dos décadas del siglo XX. En C. Cox (Ed.). Políticas educacionales en el cambio de siglo. La reforma del sistema escolar de Chile (pp.19-114). Santiago de Chile: Editorial Universitaria.

Dubet, F. (2006). El declive de la institución. Profesiones, sujetos e individuos en la modernidad. Barcelona: Gedisa.

Dubet, F (2007). El declive y las mutaciones de la institución. Revista de Antropología Social, 16, 39-66. Doi: 10.5209/rev_RASO.2007.v16.9968

Dubet, F. y Martuccelli, D. (1998). En la Escuela. Sociología de la experiencia escolar. Buenos Aires: Losada.

Durkheim, E. (1976). Educación como socialización. Salamanca: Sígueme.

Duschatzky, S. (2017). Política de escucha en la escuela. Buenos Aires: Paidós.

Duschatzky, S. y Corea, C. (2011). Chicos en banda. Los caminos de la subjetividad en el declive de las instituciones. Buenos Aires: Paidós.

Duschatzky, S., Farrán, G. y Aguirre, E. (2010). Escuelas en escena. Una experiencia de pensamiento colectivo. Buenos Aires: Paidós.

Edwards, V., Calvo, C., Cerda, A., Gómez V. e Inostroza, G. (1995) El liceo por dentro: estudio etnográfico sobre prácticas de trabajo en educación media. Santiago: Mineduc.

Espinoza, O., González, L., Castillo, D. y Neut, S. (2018). Expectativas educacionales de estudiantes que concurren a escuelas de "segunda oportunidad". Revista Mexicana de Investigación Educativa, 23(79), 1171-1193. Doi: 10.1007/978-90-481-9763-7_1

Flores, L. (2001). El sentido de la educación y las transformaciones antropológicas del mundo 
moderno. Primera aproximación al fenómeno de la violencia en el contexto escolar. Pensamiento Educativo, 28, 105-128.

Flores, L. y Sobrero, V. (2011). Subjetividad y política: consecuencias para el discurso educativo. Revista Estudios Pedagógicos, 37(2), 315-327. Doi: 10.4067/S0718-07052011000200019

Gallego, F. y Sapelli, C. (2007). El financiamiento de la educación en Chile: una evaluación. Revista Pensamiento Educativo, 40(1), 263-284. Última visita 01 de diciembre de 2018. Recuperado desde http://pensamientoeducativo.uc.cl/files/journals/2/articles/407/public/407-916-1-PB.pdf

García-Huidobro, J. (2007). Desigualdad educativa y segmentación del sistema escolar. Consideraciones a partir del caso chileno. Revista Pensamiento educativo, 40(1), 65-85.

Giruox, H. (2004). Teoría y resistencia en educación. México DF: Siglo XXI.

Guerrero, P. y Palma, E. (2010). Representaciones Sociales sobre educación de niños y niñas de calle de Santiago y Quito. Revista Latinoamericana de Ciencias Sociales, Niñez y Juventud, 8(2), 1025-1038. Última visita 01 de diciembre de 2018. Recuperado desde https://www.redalyc.org/ pdf/773/77315155018.pdf

Hevia, R. (2006). Frente a la crisis de sentido, una pedagogía de la confianza. Revista PRELAC, 2, 7075. Última visita 20 de noviembre de 2018. Recuperado desde http://www.derechoshumanos. unlp.edu.ar/assets/files/documentos/frente-a-la-crisis-de-sentido-una-pedagogia-de-laconfianza.pdf

Kessler, G. (2002). La experiencia escolar fragmentada. Estudiantes y docentes en la escuela media en Buenos Aires. Buenos Aires: IIPE-UNESCO.

Larrosa, J. (2004). La experiencia y sus lenguajes. Ponencia de serie «Encuentros y Seminarios». Última visita 30 de diciembre de 2018. Recuperado desde http://www.bnm.me.gov.ar/giga1/ documentos/EL001417.pdf

Lechner, N. (1982). La vida cotidiana en Chile: la experiencia escolar. Santiago: FLACSO. Última visita 15 de diciembre de 2018. Recuperado desde http://flacsochile.org/biblioteca/pub/ memoria/1982/001118.pdf

Lemaitre, M. (1992). Los jóvenes y la educación media. En S. Rittershaussen y J. Scharager (Eds.). Análisis y proyecciones en torno a la educación media y el trabajo (pp. 7-34). Santiago: Corporación de Promoción Universitaria.

Lewkowicz, I. (2008). Pensar sin Estado. La subjetividad en la era de la fluidez. Buenos Aires: Paidós.

Lewkowicz, I. y Corea, C. (2011). Pedagogía del Aburrido. Escuelas destituidas, familias perplejas. Buenos Aires: Paidós.

Mena, M. y Rittershaussen, S. (Eds.). (1991). La juventud y la enseñanza media: una crisis por resolver. Santiago: Corporación de Promoción Universitaria.

MINEDUC (1994). Fundamentos, estrategias y componentes del programa de Mejoramiento de la Calidad y Equidad de la Educación Media. Santiago: Ministerio de Educación.

Molina, W. (2008). Sentidos de la enseñanza media desde la experiencia escolar de estudiantes de liceos municipales. Revista Estudios pedagógicos, 34(1), 105-122. Doi: 10.4067/S071807052008000100006

Molina, W. (2013a). Sentido de Futuro en Estudiantes Secundarios: Paradojas de Equidad y Calidad desde su Experiencia Escolar. Revista Estudios pedagógicos, 39(1),143-164. Doi: 10.4067/ S0718-07052013000100009

Molina, W. (2013b). Juventudes escolarizadas, sentidos y metáforas sobre el liceo público municipal en el Chile contemporáneo. Última década, 21(38), 37-65. Doi: 10.4067/S071822362013000100003

Molina, W. y Oliva, I. (2015). Interfaces complejas en políticas educativas y de juventud: sentidos e identidades. Revista Latinoamericana de Ciencias Sociales, Niñez y Juventud, 13(2), 11251140. Doi:10.11600/1692715x.13240130114

Moya, M. (2008). El sentido de la educación formal en jóvenes urbanos populares de la comuna de Lo Espejo. Revista Iberoamericana de Educación, 46(6), 1-12. 
Estudios Pedagógicos XLV, № 1: 151-168, 2019

EL SENTIDO DE LA ESCUELA EN CHILE. LA CREACIÓN DE PARADIGMAS ANTAGÓNICOS A PARTIR DEL DISCURSO DE POLÍTICA PÚBLICA, EL DISCURSO ACADÉMICOY LA INVESTIGACIÓN EDUCATIVA

Neut, S. (2018). El conocimiento escolar en disputa en Chile: el caso del currículum fracasado de 1992. Currículo sem Fronteiras, 18(2), pp. 614-638. Última visita 1 de diciembre de 2018. Recuperado desde http://www.curriculosemfronteiras.org/vol18iss2articles/neut.pdf

Neut, P. y Miño, R. (2018). Construir la equidad desde los sujetos. Una propuesta al margen del discurso interpretativo dominante sobre la escuela. En P. Rivera, J., Muñoz, R. Morales y S. Butendieck (Eds.). Políticas Públicas para le Equidad Social (pp. 83-92). Santiago de Chile: Colección Políticas Públicas, Universidad de Santiago de Chile. Doi: 10.13140/RG.2.2.34994.50886/1

Noyola, G. (2000). Modernidad, disciplina y educación. México: Universidad Pedagógica Nacional.

Prieto, M. (2005). La participación de los estudiantes. ¿Un camino hacia su emancipación? Theoria, 14(1), 27-36. Última visita 10 de diciembre de 2018. Recuperado desde http://www.ubiobio.cl/ theoria/v/v14/a3.pdf

Quaresma, M. y Zamorano, L. (2016). El sentido de pertenencia en escuelas públicas de excelencia. Revista Mexicana de Investigación Educativa, 21(68), 275-298. Última visita 23 de diciembre de 2018. Recuperado desde http://www.scielo.org.mx/pdf/rmie/v21n68/1405-6666rmie-21-68-00275.pdf

Ravelo, M. y Radovic, Y. (2018). Representaciones de lo político en estudiantes secundarios en Santiago de Chile: resignificando el sentido de la formación ciudadana. Revista Latinoamericana de Ciencias Sociales, Niñez y Juventud, 16(1), 389-402. Doi: 10.11600/1692715x.16124

Redon, S., Angulo, J, y Vallejos, N. (2015). El Sentido de lo Común como Experiencia de Construcción Democrática. Estudio de Casos en Escuelas en Contextos de Pobreza en Chile. Archivos Analíticos de Políticas Educativas, 23(13), 1-30. Doi: 10.14507/epaa.v23.1722

Redondo, J. y Muñoz, L. (Ed.) (2009). Juventud y Enseñanza Media en Chile del Bicentenario. Antecedentes de la revolución pingüina. Santiago: OPECH. Última visita 20 de noviembre de 2018. Recuperado desde http://www.opech.cl/Libros/doc4.pdf

Rojas, X. (2006). Experiencia escolar juvenil y el ocaso de la institución escolar. Revista Castalia, $8(11), 66-79$.

Salinas, A, y Franssen, A. (1997). El zoológico y la selva. La experiencia cultural de los jóvenes de fin de siglo. Santiago: CIDE.

Sánchez, R y Santis, J. (2009). Educación, Juventud y Mundo Popular: antecedentes, perspectivas y avances de una relación distante, fragmentaria pero esperanzadora. En J. Redondo y L. Muñoz (Eds.), Juventud y Enseñanza Media en Chile del Bicentenario. Antecedentes de la revolución pingüina (pp. 439-456). Santiago: OPECH. Última visita 20 de noviembre de 2018. Recuperado desde http://www.opech.cl/Libros/doc4.pdf

Sapiains, R. y Zuleta, P. (2001). Representaciones sociales de la escuela en jóvenes urbano populares desescolarizados. Última década, 9(15), 53-72. Doi: 10.4067/S0718-22362001000200003

Sapiains, R. y Zuleta, P. (2009). Representaciones sociales de los jóvenes en situación de pobreza desescolarizados. La escuela desde la desescolarización. En J. Redondo y L. Muñoz (Eds.), Juventud y Enseñanza Media en Chile del Bicentenario. Antecedentes de la revolución pingüina (pp. 37-58). Santiago: OPECH. Última visita 20 de noviembre de 2018. Recuperado desde http://www.opech.cl/Libros/doc4.pdf

Silva, I., Bastidas, K., Calfuqueo, L., Díaz, J, y Valenzuela, J. (2013). Sentido de la Escuela para niños y niñas mapuche en una zona rural. Polis. Revista Latinoamericana, 34, 1-13. Doi: 10.4000/ polis. 7829

Tenti, E. (2000). Culturas juveniles y cultura escolar. Buenos Aires: Unesco.

Touraine, A. (2007). La mirada social. Un marco de pensamiento distinto para el siglo XXI. Barcelona: Paidós.

Valenzuela, J. (2009). Características psicométricas de una escala para caracterizar el Sentido del Aprendizaje Escolar. Universitas Psychologica, 8(1), 49-59. Última visita 28 de diciembre de 2018. Recuperado desde https://core.ac.uk/download/pdf/26957393.pdf

Willis, P. (2017). Aprendiendo a trabajar. Madrid: Akal. 